\title{
Review Article: Laboratory Findings in Covid-19
}

\author{
Hirkani Shyam Chhadi', Prajakta Warjukar² and Nandkishor Bankar \\ ${ }^{1}$ First Year MBBS Student Datta Meghe Medical College, Nagpur, India \\ ${ }^{2}$ Department of Biochemistry Datta Meghe Medical College, Nagpur, India \\ ${ }^{3}$ Department of Microbiology Jawaharlal Nehru Medical College, Datta Meghe Institute \\ of Medical Sciences Sawangi (Meghe) Wardha, India \\ Corresponding author email: drprajaktaw@gmail.com
}

\begin{abstract}
The COVID-19 epidemic first discovered in December 2019 in Wuhan, China Also known as SARS COV-2 and also associated with mild to severe respiratory disease conditions India became the second most affected by Covid 19 with 12.6 million cases and $1.6 \mathrm{k}$ death to date. Maharashtra offers the highest load status of Covid -19 in the india reporting category for all cases. It is a systemic disease involving various systems such as the heart, respiratory system, intestines, nerves, hematopoietic and immune systems. The disease exhibits variable clinical studies from mild to severe. Most patients have no symptoms, patients with comorbiditities have increased clinical decline and death and this is the group of patients we need to diagnose. Numerous studies have shown that many laboratory parameters that are easily accessible and inexpensive, can adequately predict the severity of the disease at an early age. In a country with limited resources such as India, costly investigations could not be carried out in this epidemic. It is necessary for patients to be assessed on these simple and inexpensive parameters specified in this review. During the lymphocyte count process tests a complete blood count showed that lymphopenia was a basic laboratory detection that emerged as a negative predictor factor. Neutrophil lymphocyte levels also have predictive value in determining critical cases. Some of the inflammatory symptoms including LDH, CRP, IL-6 and serum ferritin also indicate cases of malignant speculation and require immediate intervention to improve survival. Serum ferritin levels were higher in patients with severe covid-19 than in patients with non-malignant illness. D-dimer levels were consistently elevated in reported instances and were found to be associated to the severity of the disease. The most common laboratory diagnoses found in covid patients 19 increased CRP, increased D-dimer, decreased albumin, increased ESR, decreased eosinophils, increased IL6, lymphopenia and LDH. These patients need urgent care and need to be transferred to intensive care units.
\end{abstract}

KEY WORDS: SARS COV-2, COVID-19, CRP, D-DIMER.

\section{INTRODUCTION}

As of December 2019, SARS-CoV-2-linked pneumonia, called coronavirus disease 2019 (COVID-19), first appeared in Wuhan, China. The epidemic spread rapidly around the world within three months and by March

Biosc Biotech Res Comm P-ISSN: 0974-6455 E-ISSN: 2321-4007

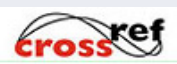

Identifiers and Pagination

Year: 2021 Vol: 14 No (7) Special Issue

Pages: 116-119

This is an open access article under Creative

Commons License Attribn 4.0 Intl (CC-BY). DOI: $h t t p: / / d x$.doi.org/10.21786/bbrc/14.7.28
11,2020 was recognized as a pandemic by WHO (Kai Duan et al., 2020).

The first case of covid-19 in INDIA was reported on January 27, 2020 and was from Trissur, Kerala. Her laboratory tests were performed on the 3rd, 7th and 20th day of the illness and the parameters were related to the infection.2 More instances were later discovered. The angiotensin converting enzyme 2 (ACE2) receptors are bound by SARS-COV-2.3 Patients with corona virus 2 (SARS-CoV-2) often show abnormal laboratory parameters. Numerous studies have shown that certain simple and inexpensive laboratory parameters can predict the severity of the disease at the onset of the disease. It is

\section{Article Information \\ Received: $10^{\text {th }}$ April 2021} ccepted after revision: $08^{\text {th }}$ June 2021 
important to investigate the effect of laboratory levels on the adverse effects of corona virus 2019 (COVID-19).

Affected laboratory levels increase active $\mathrm{C}$ protein (CRP), increase erythrocyte sedimentation rate, decrease eosinophils, increase interleukin -6, increase procalcitonine, lymphopenia, neutrophil lymphocyte ratio (NLR) thrombocytopenia, increases $\mathrm{LDH}$ and elevates D-dimer. An increase in CRP, lymphopenia, and LDH are all highly linked with severity, according to a metaanalysis of 30 studies. These data suggest that when interpreting laboratory findings in COVID-19 patients, extra caution is required. Patients with elevated CRP, lymphopenia, or LDH should be managed properly and, if necessary, referred to an intensive care unit. Studies have reported laboratory differences between those admitted to icu and standard units. High levels of white blood cell counts and neutrophils, creatinine kinase and creatinine are seen in icu patients (Wang D et al., 2020). Early identification of individuals who are at risk of becoming critically ill is vital in the treatment of this disease (Zhao Q et al., 2020). In this article we tried to show the differences of laboratory parameters of covid 19 patients with different severity and survivability.

\section{METHODS}

We examined the PubMed, Scopus, Web of Science, and Google Scholar medical databases for literature published before May 21 in order to gain a better understanding of the laboratory results in SARS cov2. The link between various laboratory test results and the severity and course of the disease was researched and assessed in 30 studies that provided a list of laboratory tests in Covid 19 patients.

\section{DISCUSSION}

As the covid 19 epidemic is spreading rapidly around the world, that is why systematic meta-analysis is urgently needed for early detection of potentially dangerous patients, their effective management and treatment to control the severity and progression of the disease. These studies have shown that the most common findings in the laboratory are increased CRP, reduced albumin, increased ESR, decreased eosinophils, increased IL6, Lymphopenia and increased LDH. SARS- CoV-2 has a high affinity for spike protein binding to angiotensin-converting enzyme 2 present in the surface of alveolar type 2 cells, kidney cells. The kidneys are one of the most important organs of the virus that causes severe kidney failure in many cases in general. In the later stages of the disease the adverse clinical condition is accompanied by a rapid increase in levels of acute phase reactants (CRP, ESR), coagulation findings and cellular confusion.

Lymphocyte and SARS-COV-2: Lympopenia is seen in the majority of instances. Lymphocytes contain ACE2 receptors on their surfaces, therefore the virus directly affects them and causes them to lyse, implying that SARSCoV2 consumes immune cells and inhibits immunological function in people (Evengelos Terpos et al., 2020). In addition cytokine storms lead to increased levels of interleukins (IL-6, IL-2, IL-7, granulocyte colony stimulating factor, etc.) and TNF alpha causing lymphocyte apoptosis. damages lymphocyte gains. 3 Lympopenia is linked to a higher risk of ARDS. Increased neutrophils and lymphopenia have been linked to the severity of sickness in COVID-19 patients in several investigations (Chen T et al., 2019).

Platelets and SARS-CoV-2: Thrombocytopenia has been observed in rare situations. The severity of covid -19 illness is strongly linked to thrombocytopenia. Due to increased platelet activation, a high platelet to lymphocyte ratio may signal a more intense cytokine storm. Thrombocytopenia is seen in 5-41.7 percent of COVID-19 patients, and it is usually modest (counts range from 100 to 150 times 109/L). The platelet count of patients with severe illness is only $23 \times 109 / \mathrm{L}$ to 31 $\mathrm{x}$ 109/L lower than that of those with mild illness. 7, 16-17 In COVID-19 individuals, for example, severe thrombocytopenia has only been documented seldom in combination with an immune thrombocytopenic purpura-like condition.

CRP and SARS-COV-2: CRP is a important biochemical inflammatory marker, severe cases are associated with raised CRP. 7 Higher CRP indicates viral induced cytokine storm , and has been linked to unfavourable aspects like ARDS development. CRP has a normal concentration of less than $6 \mathrm{mg} / \mathrm{L}$, rises fast within 6-8 hours, and peaks 48 hours after the commencement of the disease. It has a half-life of around 19 hours and declines in concentration as the patient heals. CRP levels of 20 to $50 \mathrm{mg} / \mathrm{L}$ have been found in people with COVID-19 in various studies. CRP levels were reported to be up to 86 times higher than normal in severe COVID-19 patients8,19,20. Patients with severe illness courses had significantly higher CRP levels than mild or non-severe patients. According to one research, individuals with more severe symptoms had an average CRP concentration of $39.4 \mathrm{mg} / \mathrm{L}$, whereas those with minor symptoms had an average CRP concentration of $18.8 \mathrm{mg} / \mathrm{L}$. CRP levels were observed to be higher in the severe group than in the mild group at the outset.

In another study, the mean CRP concentration in severe patients $(46 \mathrm{mg} / \mathrm{L})$ was substantially greater than in non-severe patients (23 mg/L). CRP levels were 10 times greater in COVID-19 individuals who died than in those who survived (median 100 vs $9.6 \mathrm{mg} / \mathrm{L}$ ).8,21,22 According to a recent study, roughly $7.7 \%$ of non-severe COVID-19 patients developed severe disease courses after being admitted to the hospital, and the aggravated patients had significantly higher CRP concentrations (median 43.8 vs $12.1 \mathrm{mg} / \mathrm{L}$ ) than the non-severe cases. CRP levels were found to have a significant connection with non-severe COVID-19 patients' deterioration. CRP was presented as a suitable marker for predicting the aggravation probability of non-severe COVID-19 patients by 1 and the authors, with an appropriate threshold value of $26.9 \mathrm{mg} / \mathrm{L} .7,21,22$ In patients with COVID-19, every one-unit rise in CRP concentration increases the probability of experiencing 
severe episodes by 5\%, according to the authors.

LDH and SARS-COV-2: LDH levels were significantly higher in severe patients than in non severe patients. 7 LDH levels are known to be increased in obstructive lung disease and microbial pulmonary disease. 8,9 Multiple organ damage and failure appear to have a more important role in this pathophysiology in impacting clinical outcomes in COVID-19 individuals with elevated LDH levels. In thrombotic microangiopathy, which is linked to renal failure and cardiac damage, LDH levels are also high. Severe infections may result in tissue damage mediated by cytokines and the production of $\mathrm{LDH}$. Because LDH is present in lung tissue (isozyme 3), patients with severe COVID-19 infections should anticipate to release more LDH into the blood, since the condition is characterised by a severe type of interstitial pneumonia that typically progresses to acute respiratory distress syndrome. There was a $>6$-fold increase in the odds of severe illness and a $>16$-fold increase in the chances of mortality in individuals with increased LDH, according to many studies. In three studies, nonsurvivors had higher LDH levels than survivors, with >95 percent of non-survivors having higher LDH levels than survivors.

D-dimer and SARS-COV-2: Patients with communityacquired pneumonia have higher D-dimer levels, which are linked to difficulties and poor outcomes. D-dimer testing is strongly recommended in covid 19 patients, according to recently published guidelines. In patients with increased D-dimer levels, the SARS CoV-2 trial found a substantial link between acute and covid impact 19. Increased D-dimer levels imply coagulation and fibrinolytic adherence, both of which necessitate ongoing diagnostic and treatment methods. On the other hand, low-dose DDimer can be used to rule out venous thrombotic (VTE) events such as venous thrombosis (DVT) and pulmonary embolism (PE). (ILI) due to the effectiveness of coagulation with respiratory bacteria .Tests are often used as part of a diagnostic algorithm to rule out a diagnosis of thrombosis. Patients with severe COVID-19 infections have higher D-dimer levels than those with mild infections, and D-dimer levels more than $0.5 \mathrm{~g} / \mathrm{ml}$ are linked to severe infection in COVID19 patients.

IL-6 and SARS-COV-2: Patients with high IL-6 levels are at a higher risk of developing respiratory failure. In patients who needed to be ventilated, IL-6 levels were considerably higher.

LFT and KFT: Other side effects of COVID-19 patients include parameters to assess liver and kidney function. The majority of patients had high concentrations of alanine aminotransferase, aspartate aminotransferase, amount of bilirubin, lactate dehydrogenase, alkaline phosphatase and $\gamma$-glutamyl transpeptidase and were significantly higher in patients who died compared to obese patients. On the other hand, hypoalbuminemia has been shown in COVID-19 patients. The concentration of urea nitrogen in the blood, ferritin, potassium and triglycerides was significantly increased in all COVID19 patients, but was higher in obese patients than in patients diagnosed.11, 12 In China, a research found that died COVID-19 patients had considerably lower levels of thyroid stimulating hormone and free triiodothyronine than recovered COVID-19 patients. 11 Many studies on various aspects of Covid-19 and it's clinical implications were reported. Godhiwala et. al. reported about leukemoid reaction in a covid-19 patient. Articles on best practices and guidelines in different clinical specialties were reviewed.

\section{CONCLUSION}

Elevated CRP, elevated D- dimer, decreased albumin, elevated ESR, decreased eosinophils, increased IL6, lymphopenia, and increased LDH were the most prevalent laboratory results in covid 19 individuals. These individuals require immediate care and should be transferred to intensive care units.

\section{REFERENCES}

Arora, Devamsh, Muskan Sharma, Sourya Acharya, Samarth Shukla, and Neema Acharya (October 26, 2020). "India in 'Flattening the Curve' of COVID19 Pandemic - Triumphs and Challenges Thereof." Journal Of Evolution Of Medical And Dental SciencesJemds 9, no. 43: 3252-55. https://doi.org/10.14260/ jemds/2020/713.

Ballou SP, Kushner I (1992). C-reactive protein and acute phase response. Adv Intern Med;37:? 336.

Bawiskar, Nipun, Amol Andhale, Vidyashree Hulkoti, Sourya Acharya, and Samarth Shukla (November 16, 2020). Haematological Manifestations of Covid-19 and Emerging Immunohaematological Therapeutic Strategies." Journal of Evolution Of Medical And Dental Sciences-Jemds 9, no. 46: 3489-94. https://doi. org/10.14260/jemds/2020/763.

Bidkar, Vijay, Kalaiselvi Selvaraj, Meena Mishra, Vishal Shete, and Anita Sajjanar (April 2021). A Comparison of Swab Types on Sample Adequacy, Suspects Comfort and Provider Preference in COVID-19." American Journal Of Otolaryngology 42, no. 2. https://doi.org/10.1016/j. amjoto.2020.102872.

Bomhof G, Mutsaers PG, et al (2020 Jul). COVID-19associated immune thrombocytopenia. Br J Haematol; 190(2):page61-4.

Chen N, Zhou M, Dong X, et al. Epidemiological and clinical characteristics of 96 cases of 2019 novel coronavirus pneumonia in China: a descriptive study. The Lancet. - 1016/S0140-

Chen T, Wu D, Chen H et al (2020). Clinical characteristics of 113 deceased patients with coronavirus disease 2019: retrospective study. BMJ 368, m1091.

Chen T, Wu D, Chen H et al (2020). Clinical characteristics of 113 deceased patients with coronavirus disease 2019: retrospective study. BMJ 368, m1091.

Evengelos Terpos (2020), Ioannis Ntanasis-Sthathopoulos, et al. Hematological findings and complications of 
COVID-19.DOI:10.1002/ajh.25829.

Godhiwala, Parth, Sourya Acharya, Gaurav Jagtap, Arvind Bhake, and Samarth Shukla (February 8, 2021). Leukemoid Reaction in a COVID-19 Patient." Journal Of Evolution Of Medical And Dental Sciences-Jemds 10, no. 6: 399-400. https://doi.org/10.14260/jemds/2021/88. Henry BM, de Oliveira MH, Benoit S, Plebani M, Lippi G (2020 Jun). Hematologic, biochemical and immune biomarker abnormalities associated with severe illness and mortality in coronavirus disease 2019 (COVID-19): a meta-analysis. Clin Chem Lab Med; 58(7): 1021-8.

Huang C, Wang Y, Li X et al (2020). Clinical features of patients infected with 2019 novel coronavirus in Wuhan, China. Lancet 395(10223), 497-506.

Inamura N. , Miyashita N. , Hasegawa S., et al. Management of refractory Mycoplasma pneumoniae pneumonia: Utility of measuring serum lactate dehydrogenase level.DOI:https://doi.org/10.1016/j. jiac.2014.01.001.

Kai Duan, Bende Liu, Cesheng Li , et al. Effectiveness of convalescent plasma therapy in severe COVID-19 patients.PNAS. April 28,2020.vol 117,no.17.

Khubchandani, Sheetal Rameshlal, and Trupti Madhav Dahane (October 12, 2020). Emerging Therapeutic Options for COVID-19.” Journal Of Evolution Of Medical And Dental Sciences-JEMDS 9, no. 41: 3082-85. https:// doi.org/10.14260/jemds/2020/677.

Kute, Vivek, Sandeep Guleria, Jai Prakash, Sunil Shroff, Narayan Prasad, Sanjay K. Agarwal, Santosh Varughese, et al (August 2020). "NOTTO Transplant Specific Guidelines with Reference to COVID-19.” Indian Journal Of Nephrology 30, no. 4: 215-20. https://doi. org/10.4103/ijn.IJN_299_20.

Lippi G, Plebani M, Henry BM (2020 Jul). Thrombocytopenia is associated with severe coronavirus disease 2019 (COVID-19) infections: A meta-analysis. Clin Chim Acta; 506:145-8.

MA Andrews, Binu Areekal, KR Rajesh, et al (2020). First confirmed case of COVID-19 infection in India: A case report. Year,Volume : 151, Issue : 5 , Page : 490-492.

Mo P.,Xing Y. ,Xiao Y. , et al (2020). Clinical characteristics of refractory COVID-19 pneumonia in Wuhan, China.March Clinical Infectious Diseases. DOI:10.1093/cid/ciaa270.
P. Sabaka, A. Košðálová2, I. Straka3, MC Infectious Diseases (2021) 21:308 https://doi.org/10.1186/s12879021-05945-8.

Pate, Bhavna Shrirang, Meenakshi Eknath Yeola, Atul Gawande, Amit Kumar Singh, and Harshal Atul Tayade (December 7, 2020). "Best Practices for Endoscopic Procedures in Covid-19 Pandemic." JOURNAL OF EVOLUTION OF MEDICAL AND DENTAL SCIENCESJEMDS 9, no. 49: 3760-66. https://doi.org/10.14260/ jemds/2020/825.

Pepys MB, Hirschfield GM (2003). C-reactive protein critical update. J Clin Invest;111(12):1805- 1812. 10.1172/JCI200318921

Tobias Herold, Vindi Jurinovic, Chiara Arnreich , et al. Level of IL- 6 predicts respiratory failure in hospitalized symptomatic COVID-19 patients. Journal of Allergy and Clinical Immunology doi: 10.1016/j.jaci.2020.05.008. Wang D, Hu B, Hu C et al (2020). Clinical characteristics of 138 hospitalized patients with 2019 novel coronavirusinfected pneumonia in Wuhan, China. JAMA 323, 1061-1069.

Yang X, Yang Q, Wang Y, Wu Y, Xu J, Yu Y, et al (2020 Jun). Thrombocytopenia and its association with mortality in patients with COVID-19. J Thromb Haemost; 18(6): 1469-72.

Young B, Gleeson M, Cripps AW. C-reactive protein: a critical review.Pathology.1991;23(2):118-124. 10.3109/003130291090608

Zachary Illg, D0 Gregory Muller, D0, Matthew Mueller, DO, MPH ((2021)), Analysis of absolute lymphocyte count in patients with COVID-19American Journal of Emergency Medicine 46;16-19

Zhang Y, Zeng X, Jiao Y, Li Z, Liu Q, Ye J, et al (2020 Sep). Mechanisms involved in the development of thrombocytopenia in patients with COVID- 19. Thromb Res; 193:110-5.

Zhao Q ,Meng M, Kumar R ,Wu Y, Huang J ,Lian N ,et al. The impact of COPD and smoking history on the severity of COVID-19: A systemic review and metaanalysis.J Med Virol.2020;n/a.

Zu-Li Zhang, Yu-Lei Hou, Feng-Zeng Li (May 2020). Laboratory findings of COVID-19: a systematic review and meta analysis.Scandinavian Journal Of Clinical And Laboratory Investigation. 